\title{
The effect of Allium sativum in experimental peritoneal adhesion model in rats ${ }^{1}$
}

\author{
Uğur Topal' (D) , Nuri Emrah Göret" (D) , Ceren Canbey Göret"II (D) , Ömer Faruk Özkanv
}

' MD, Department of General Surgery, Erciyes University Medical Faculty, Melikgazi, Kayseri, Turkey. Conception and design of the study, statistics analysis, manuscript preparation and writing, final approval.

" MD, Department of General Surgery, Health Sciences University, Kartal Dr Lütfi Kırdar Research and Education Hospital, Istanbul, Turkey. Acquisition of data, technical procedures, final approval.

II'MD, Department of Surgical Pathology, Health Sciences University, Sancaktepe Research and Education Hospital, Istanbul, Turkey. Conception and design of the study, analysis and interpretation of data, technical procedures, histopathological examinations, manuscript writing, final approval.

Iv MD, Department of General Surgery, Health Sciences University, Ümraniye Research and Education Hospital, Istanbul, Turkey. Acquisition, analysis and interpretation of data, technical procedures, statistics analysis, manuscript writing, critical revision, final approval.

\begin{abstract}
Purpose: To evaluate the effect of garlic on formation of postoperative adhesions in rats.

Methods: Twenty-four Sprague dawley rats were divided into three groups. In Group 1 (sham), laparotomy was performed and stitched up. In Group 2 (control), after laparotomy was performed, punctate hemorrhage was induced by cecal abrasion in the cecum and $2 \mathrm{cc}$ of saline was intraperitoneally administered to each rat. In Group 3 (experimental), after laparotomy was performed, punctate hemorrhage was induced by cecal abrasion in the cecum and each rat was intraperitoneally administered a sterile Allium sativum derivative. The rats in all groups were re-laparotomized on postoperative day 7; samples were obtained from the peritoneal tissue surrounding the cecum

Results: In Group 3, there was a statistically significant difference in terms of inflammation, lymph node size, and free oxygen radicals; these parameters tended to increase. In terms of fibrosis evaluated using H\&E and MT, there was no significant difference between groups 2 and 3.

Conclusions: No positive outcomes indicating that Allium sativum reduces intra-abdominal adhesions were obtained. However, it caused severe inflammation in the tissue. Additionally, in immunohistochemical analyses conducted to detect oxidative stress, allium sativum increased the production of free oxygen radicals in the tissue.
\end{abstract}

Key words: Garlic. Lymphadenopathy. Free Radicals. Rats. 


\section{- Introduction}

Postoperative peritoneal adhesions (PA), defined as fibrous band formation between intra-abdominal organs, is one of the most important problems of surgery. It is stated that the frequency of PA increases up to $90 \%$ in some sources ${ }^{1}$. Abdominal reoperation is more difficult and riskier when there are adhesions. Increased enterotomy risk, longer dissection times and longer operation times are serious consequences of intraabdominal adhesions ${ }^{2,3}$.

PAs are caused by the normal peritoneal healing not being limited, due to an increase in vascular permeability as a result of peritoneal damage and release of fibrinrich exudate. Various drugs, medical agents and surgical techniques have been tried to prevent PPA formation, but there is currently no active and accepted treatment modality. Experimental animal studies are preferred for the studies on prevention of PPAs, mainly because it is difficult and risky to apply to humans, and most of these studies are performed on rats ${ }^{4-7}$.

Inflammation and infection secondary to intraabdominal operations may cause adhesions and may be responsible for the etiopathogenesis of lymphadenopathy secondary to inflammation ${ }^{7,8}$. In animal models, many products have shown to reduce postoperative adhesion formation, such as corticosteroids, non-steroidal anti-inflammatory drugs, reactive oxygen species (ROS) scavengers, fibrinolytic agents, flotation agents and semisolid barriers and mechanical barriers ${ }^{8-11}$.

Allium sativum has been used as a medicine in traditional medicine for centuries and is a well-known substance with anti-inflammatory, antibacterial, fibrinolytic and wound healing properties ${ }^{8,12}$. Considering that if allium sativum has an effect on preventing peritoneal adhesion, it can be used in routine practice because it can be found cheap and easy to prevent postoperative adhesion, in addition to examining its effectiveness in preventing intraabdominal adhesions, we aimed to investigate the effects of free oxygen radicals immunohistochemically and the number and size of reactive lymph nodes.

\section{- Methods}

\section{Experiment protocol}

This study was performed in the laboratory of Hamidiye Turkey, with the approval of the university's Laboratory Animals Ethics Committee (No: 15.05.2019/2019-05/07).

Rats were obtained from SBU experimental animals center and kept in special cages under appropriate feeding conditions during the study. The rats were housed in special metal cages with controlled heat (between 19-22C) and illumination (08-20 light, 20-08 dark). Rats had normal water and standard foods access without restriction. Prior to the test procedure, all rats were weighed with an analytical balance and their body weights (BW) recorded. Sprague dawley rats weighing 250-300g were divided into three groups ( $n=8$, in each group).

All rats were anesthetized with intramuscular (IM) ketamine (Ketalar $500 \mathrm{mg}, 35 \mathrm{mg} / \mathrm{kg}$ body weight [BW]; Pfizer) and xylazine (Kepro Xylazine 20, $15 \mathrm{mg} / \mathrm{kg}$ BW; Biopharm Veterinary Drugs). After anesthesia, they were cleaned with $10 \%$ povidone-iodine solution. A $3 \mathrm{~cm}$ median laparotomy was performed. After the procedure, the incision was closed with continuous 3-0 polypropylene sutures (Prolene; Bicakcilar Co., Istanbul, Turkey).

Group 1 (sham): After laparotomy, the abdomen of the rats was closed again without performing any procedure. On postoperative day 7 , rats were relaparotomized under anesthesia; peritoneal tissue surrounding the cecum was sampled and rats were sacrificed.

Group 2 (control group: physiological saline solution): After laparotomy in rats, abrasion, punctate hemorrhage with sterile gauze was created in cecal fat tissue and intraperitoneal $2 \mathrm{cc}$ saline solution was applied. On postoperative day 7 , rats were relaparotomized under anesthesia; peritoneal tissue surrounding the cecum was sampled and rats were sacrificed (Fig. 1).

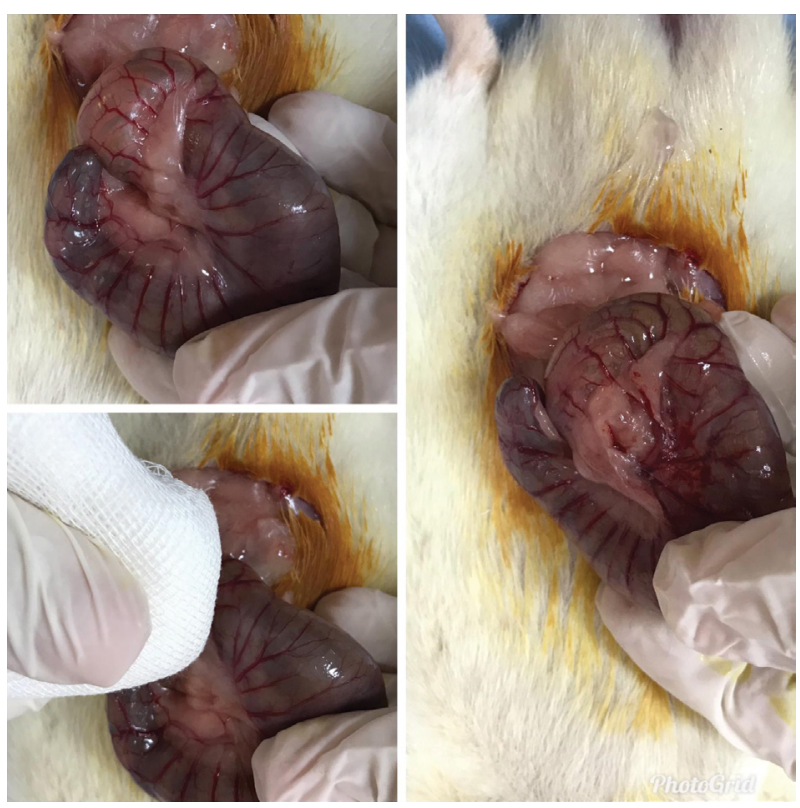

Figure 1 - Creation of punctate hemorrhage in the cecum by cecal abrasion after laparotomy. 
Group 3 (experiment group: allium sativum): After laparotomy in rats, abrasion, punctate hemorrhage with sterile gauze was created in cecal fat tissue and intraperitoneal $5 \mathrm{ml} / \mathrm{kg}$ (OleumAlliiSativiR;Arifoglu, Firuzkoy Esenyurt Road No. 32 Avcilar, Istanbul) derivative was applied. On postoperative day 7 , rats were relaparotomized under anesthesia; peritoneal tissue surrounding the cecum was sampled and rats were sacrificed.

\section{Histopathological examination}

All materials were fixed with $10 \%$ buffered formaldehyde. Samples embedded in paraffin blocks were slit sections of 4-5 micron thickness and stained with hematoxylin \& eosin (H\&E). In addition to $H \& E$, the samples were sectioned with a thickness of 3-4 microns and stained with Masson's trichrome (MT) histochemically. In addition, 3 micron sections were taken from paraffin blocks and immunohistochemical staining with GSTP1, GLUT-red, SOD1, CAT was performed using LEICA kits in LEICA automatic staining device.

Histopathological effects were evaluated according to the parameters listed below (Table 1):

Table 1 - Histopathological scoring table.

\begin{tabular}{lcccc} 
Score & Fibrosis & Inflammation & Masson Tricrom & $\begin{array}{c}\text { SOD1, CAT, GSTP1, GLUT RED } \\
\text { (Immunohistochemistry) }\end{array}$ \\
\hline 0 & None & None & None & None \\
1 & Mild & Mild & Mild & Mild \\
2 & Significant & Significant & Significant & Intense \\
3 & Intense & Intense & Intense & \\
\hline
\end{tabular}

-Fibrosis

-Inflammation

-Number of lymph nodes

-Size of largest lymph node $(\mathrm{mm})$

Fibrosis Scores with H\&E were evaluated as:

No effect: 0

Light effect: 1

Moderate effect: 2

Intense effect: 3

Immunohistochemical staining scores for GSTP1, SOD1, CAT and Glut Red were evaluated as:

0 : no staining

1: focal weak positivity

2: moderate positivity

3: intense strong positivity

Histochemical fibrosis evaluation scores with Masson Trichrome (MT) were evaluated as:

0 : no staining

1: focal weak positivity

2: moderate positivity

3: intense strong positivity

\section{Statistical analysis}

Data were analyzed by SPSS 24 IBM SPSS Statistics for Windows, version 24 (IBM Corp., Armonk, N.Y.,
USA). The normal distribution of the variables was examined by analytical methods (Kolmogorow Smirnow test) considering visual (histogram and probability graphs) and sample size. Kruskal-Wallis test was used for comparisons between the groups since not all variables had normal distribution. Descriptive statistics are given as arithmetic mean \pm standard deviation and median (quarters). OneWay ANOVA test was used for binary comparisons. Differences between the groups and changes in the number of lymph nodes over time and total score variables were analyzed by two-way variance analysis. Bonferroni test was used for differences between the groups. Statistical significance level was accepted as $\mathrm{P}<0.05$.

\section{- Results}

From statistical analyses, the results of specimens in Groups 1, 2, and 3 were not normally distributed; therefore, they were compared using the KruskalWallis test.

In Kruskal-Wallis analysis, there was statistical difference between the groups in terms of inflammation, fibrosis, lymph node size and histochemical fibrosis. (Fig. 2). They were higher in Group 3 compared to other groups $(p<0.05)$ (Table 2$)$. 
In the paired group analysis, there was a statistical significance for fibrosis when Group 1 and Group 2 $(p=0.018)$ and Group 1 and Group $3(p=0.003)$ were compared, whereas when Group 2 and Group 3 were compared, it was not statistically significant $(p=0.483)$ (Table 3).

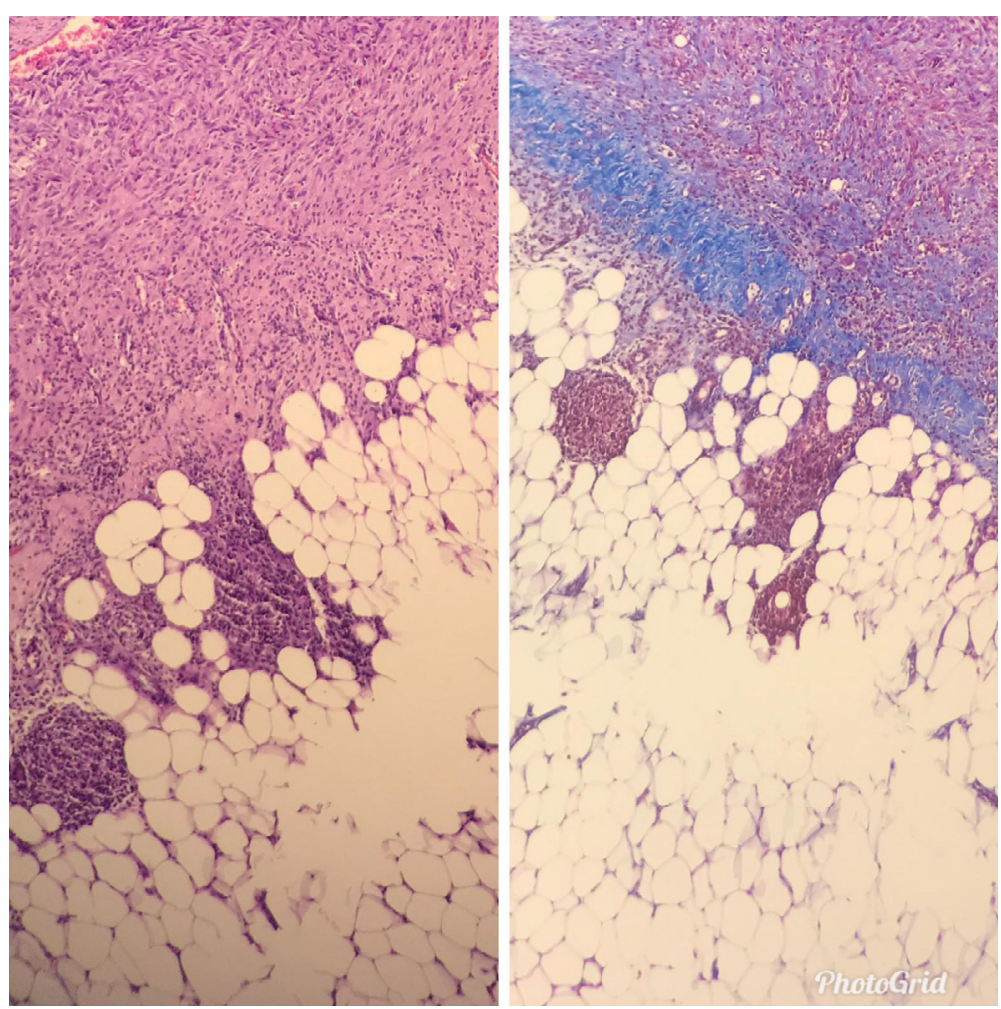

Figure 2 - On the left, Score 3 fibrosis, intense (score 2) inflammation with hematoxylin \& eosin (H\&E x100). On the right, Intense (score 3) fibrosis with Masson's Trichrome (Histochemistry x100).

Table 2 - Kruskal wallis test analysis.

\begin{tabular}{|c|c|c|c|c|c|c|c|c|c|c|c|c|}
\hline & \multicolumn{3}{|c|}{ Fibrosis } & \multicolumn{3}{|c|}{$\begin{array}{l}\text { Fibrosis with Masson's } \\
\text { trichrome }\end{array}$} & \multicolumn{3}{|c|}{ Inflammation } & \multicolumn{3}{|c|}{ Lymph node size } \\
\hline & $\begin{array}{l}\text { Mean } \\
\text { Rank }\end{array}$ & $x^{2}$ & p* & $\begin{array}{l}\text { Mean } \\
\text { Rank }\end{array}$ & $x^{2}$ & p* & $\begin{array}{l}\text { Mean } \\
\text { Rank }\end{array}$ & $x^{2}$ & p* & $\begin{array}{l}\text { Mean } \\
\text { Rank }\end{array}$ & $x^{2}$ & p* \\
\hline Group 1 & 6.50 & 9.088 & 0.011 & 6.88 & & 0.011 & 6.75 & & 0.009 & 6.50 & & \\
\hline Group 2 & 15.44 & & 14.19 & & 8.979 & 13.69 & & 9.503 & 15.44 & & 9.088 & 0.011 \\
\hline Group 3 & 15.56 & & 16.44 & & & 17.06. & & & 15.56 & & & \\
\hline
\end{tabular}

Table 3 - Histopathological evaluation of the study groups.

\begin{tabular}{lccc} 
Groups & Fibrosis & $\begin{array}{c}\text { Fibrosis with Masson's } \\
\text { trichrome }\end{array}$ & Inflammation \\
\hline Group 1-2 & P $0.018^{*}$ & P $0.018^{*}$ & P 0.026* \\
Group 1-3 & P $0.003^{*}$ & P $0.003^{*}$ & P $0.003^{*}$ \\
Group 2-3 & P 0.483 & P 0.483 & P $0.0 .008^{*}$ \\
\hline
\end{tabular}

$* P<0.05$, pair-group analysis was evaluated via the Mann-Whitney $U$ test.

**Group 1: sham group, group 2: control group, group 3: experimental group.

*** Lymph node size was microscopically measured in the histopathological examination in $\mathrm{mm}$. 
For fibrosis with Masson's Trichrome, there was a statistical significance when Group 1 and Group 2 $(p=0.018)$ and Group 1 and Group $3(p=0.003)$ were compared, whereas when Group 2 and Group 3 were compared, it was not statistically significant $(p=0.483)$.

For inflammation, there was a statistical significance when Group 1 and Group $2(p=0.026)$ and Group 1 and Group 3 ( $p=0.003$ ) were compared, whereas when Group 2 and Group 3 were compared, it was not statistically significant ( $p=0.179)$.

For lymph node size, there was a statistical significance when Group 1 and Group $2(p=0.01)$ and Group 1 and Group 3 ( $p=0.007$ ) were compared, whereas when Group 2 and Group 3 were compared, it was not statistically significant $(p=1.000)$.
The total immunohistochemical score and number of lymph nodes were normally distributed, and statistical evaluation between the three groups was done using one-way ANOVA.

When the groups were compared in terms of immunohistochemical score, the mean values were 1.75 in Group 1, 5.25 in Group 2, and 4.33 in Group 3. The $P$-value was $0.0001(P<0.005)$, and there was a significant difference (Table 4).

In the paired group analysis in terms of immunohistochemical score, there was a statistical significance between Group 1 and Group $2(p=0.004)$ and Group 1 and Group 3 ( $p=0.001)$, whereas the difference between Group 2 and Group 3 was not statistically significant $(p=1.000)$ (Table 5).

Table 4 - Results of comparison with immunohistochemical score groups.

\begin{tabular}{|c|c|c|c|c|c|}
\hline & \multicolumn{3}{|c|}{ Groups } & \multirow{2}{*}{$\mathbf{F}$} & \multirow{2}{*}{$\mathbf{p}$} \\
\hline & Group A & Group B & Group C & & \\
\hline Immunohistochemical score & $\begin{array}{c}1.75+2.37 \\
0.0-7.0\end{array}$ & $\begin{array}{c}5.25+1.03 \\
4.0-7.0\end{array}$ & $\begin{array}{c}6.0+2.07 \\
4.0-9.0\end{array}$ & 11.227 & 0.000 \\
\hline
\end{tabular}

Each immunohistochemical marker was individually scored, but statistical evaluation was performed for each rat on the basis of the total score
(SOD-1, CAT, GSTP-1, Glut Red). Statistical evaluation between the three groups was done using one-way ANOVA.

Table 5 - Comparison between the groups with respect to the immunohistochemical score and the number of lymph nodes.

\begin{tabular}{lcc} 
Groups & Number of lymph nodes & Immunohistochemical score \\
\hline Group 1-2 & 1.000 & $0.004^{*}$ \\
Group 1-3 & 1.000 & $0.001^{*}$ \\
Group 2-3 & 1.000 & 1.000 \\
\hline
\end{tabular}

*The mean difference is significant at the level of 0.15 .

** Post hoc pair-group analysis was performed using Bonferroni correction.

*** Statistical evaluation between the three groups was assessed with one-way ANOVA.

**** Each immunohistochemical marker was individually scored, but statistical evaluation was performed for each rat on the basis of the total score (SOD-1, CAT, GSTP-1, Glut Red).

$* * * * *$ In the histopathological examination, the number of lymph nodes was microscopically counted for each rat.

In immunohistochemical analyses (SOD-1, Glut Red, CAT, GSTP-1) on free oxygen radicals, allium sativum statistically increased the production of free oxygen radicals in the tissues (Fig. 3 ).
In terms of the number of lymph nodes, the mean values were 3.1111 in Group 1, 3.6667 in Group 2, and 1.4444 in Group 3. P value was 0.934 ( $P>0.005$ ) and there was no statistically significant difference (Table 6). 

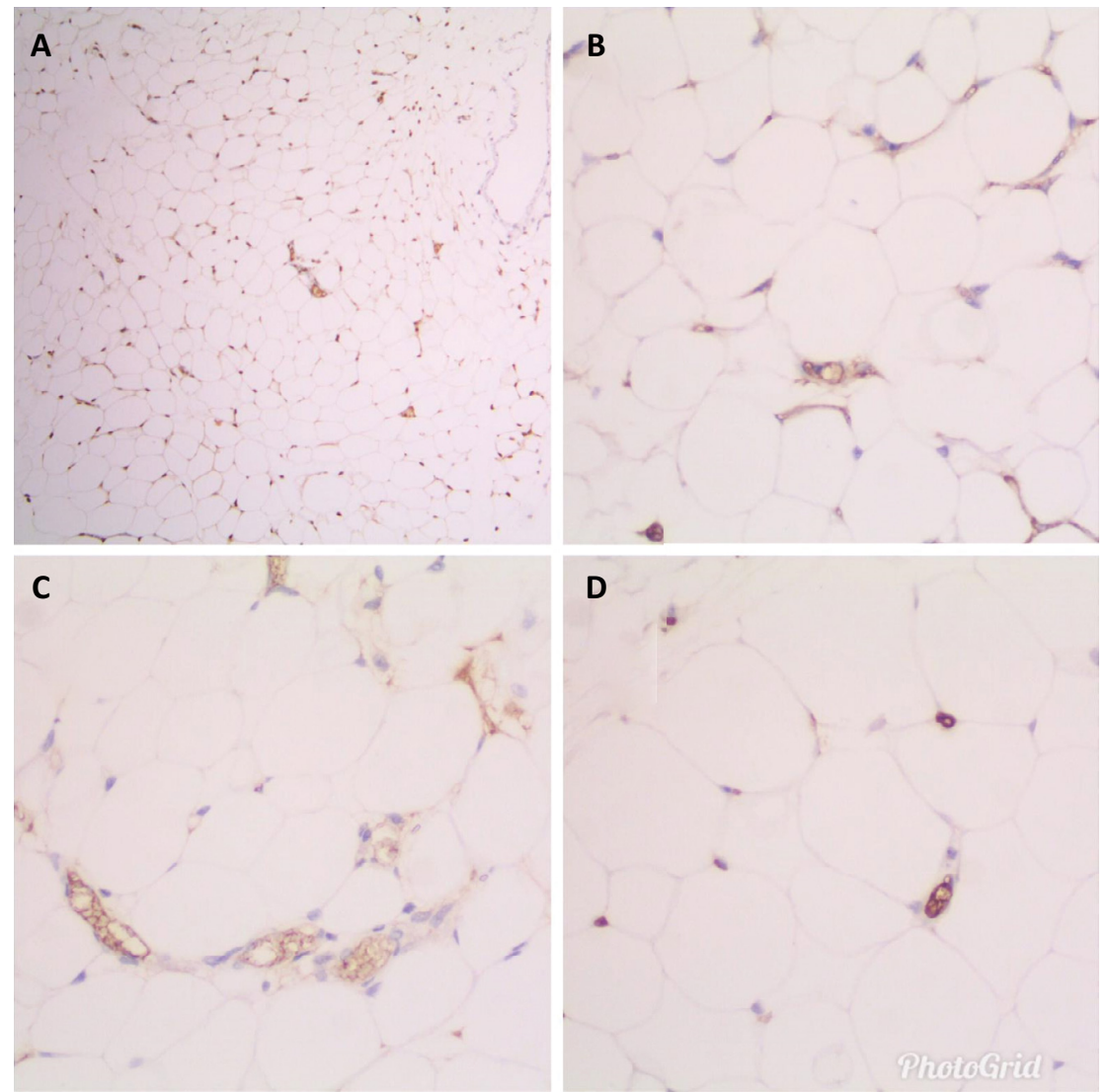

Figure 3 - A. Intense (Score 3) staining of adipose tissue with SOD 1 (IHC x40). B. Moderate positivity (Score 2) with GSTP 1 (IHC x400). C. Moderate positive (Score 2) staining with CAT (IHC x400). D. Light (Score 1) positive staining with GLUT RED (IHC x400).

Table 6 - Mean values of free oxygen radicals number of lymph nodes.

\begin{tabular}{ccc} 
Groups & $\begin{array}{c}\text { Number of lymph } \\
\text { nodes }\end{array}$ & $\begin{array}{c}\text { Free oxygen } \\
\text { radicals with } \\
\text { immunohistochemical }\end{array}$ \\
\hline Group 1 & St. deviation: 1.923 & St. deviation: 2.37547 \\
Median: 4 & Median: 1.00 \\
Group 2 & St. deviation: 1.753 & St. deviation: 1.03510 \\
& Median: 4 & Median: 5 \\
Group 3 & St. deviation: 2.449 & St. deviation: 2.63202 \\
& Median: 3.50 & Median: 4.50 \\
\hline
\end{tabular}

*The mean difference is significant at the level of 0.15 .

** Post hoc pair-group analysis was performed using Bonferroni correction.

*** Statistical evaluation between the three groups was assessed with one-way ANOVA.

**** Each immunohistochemical marker was individually scored, but statistical evaluation was performed for each rat on the basis of the total score (SOD-1, CAT, GSTP-1, Glut Red). $* * * * *$ In the histopathological examination, the number of lymph nodes was microscopically counted for each rat.
In the paired group analysis for the number of lymph nodes, the $P$ value was 1.0 between Groups 1 and 2, 1.0 between Groups 1 and 3, and 1.0 between Groups 2 and 3 (Table 5).

Post-hoc paired group analyses were evaluated using Bonferroni correction $* * *$ for total immunohistochemical score and number of lymph nodes (Mean difference was significant at 0.15.).

\section{- Discussion}

Peritoneal adhesions (PA) remain a major health problem and, according to some sources, have been reported to be seen at very high rates, especially after abdominal surgery ${ }^{1}$. Intestinal obstruction, abdominal pain due to visceral peritoneal traction, infertility due to extraluminal compression, urinary dysfunction due to traction of ureters may be seen secondary to $\mathrm{PA}^{13}$.

PA may be congenital or especially acquired in the postoperative period ${ }^{13}$. In a postmortem study, approximately $1 / 3$ of the cases with peritoneal 
adhesion were found to be non-surgical cases and endometriosis, peritoneal dialysis and radiotherapy were among the events that triggered intraabdominal inflammation, but the majority of cases were reported in the postoperative period ${ }^{14}$.

In case of a possible re-operation in PA cases, prolongation of operation and dissection times and increased risk of surgical complications can be mentioned ${ }^{2,3}$.

Multiple strategies have been proposed to eliminate $\mathrm{PA}$, but there are varying results ${ }^{15}$.

After any intervention in the peritoneal tissue (surgery, inflammation of the trauma, infection or placement of foreign bodies in the peritoneal cavity, etc.), the development of adhesion may begin.

After injury/damage to the mesothelial cells covering the peritoneal surface, the healing process begins. Vasoactive amines such as histamine and quinine contribute to the accumulation of fibrin-rich exudates around the damaged area by increasing vascular permeability.

As the fibrin polymers in the exudate interact with the fibronectin to form the fibrin gel matrix, which produces fibrin bands between the injured area, simultaneous fibrinolytic activity begins. Fibrinolytic activity is more dominant in areas where healing occurs without adhesion. In contrast, if there is a defect or imbalance in fibrinolytic activity, there is persistence of fibrinous material in these areas. Secondary to this, proliferative fibroblasts migrate to this area and accumulate extracellular matrix material that contains collagen that contributes to adhesion formation. Thus, the different mechanical steps, some of which are described above, regulate the healing process and the presence of imbalances in any of them potentially contributes to the development of adhesion ${ }^{7,16}$.

Free oxygen radicals are molecules that are released from polymorphic leukocytes and macrophages and show their effects in the early stage of inflammation. Free radicals target DNA, proteins and lipids, attack membrane lipids and cause peroxidation. This causes damage to the cell membranes and in this case increases the formation of microvascular edema. In recent years, it has been shown that oxygen-derived free radicals and metabolites play a role in increased cell and tissue damage in leukocyte-dependent inflammatory reactions and that these radicals cause secondary damage in many tissues $^{17,18}$. As a result, inflammation and fibrosis are observed histopathologically on a microscopic level.

Adhesions have shown to be a result of incorrect healing caused by injured peritoneal tissues associated with oxidative stress. In addition to parameters such as fibrosis and inflammation, GSTP-1, GLUT Red, SOD-1 and CAT were used to determine oxidative stress parameters immunohistochemically in tissues. On postoperative day 7 , it was reported that these adhesive strips progressed to permanent fibrous adhesions, and that hypoxia directly promoted the production of free oxygen radicals in tissues ${ }^{7,8}$.

Various antioxidant agents have been used to prevent adhesion formation by preventing tissue damage caused by free oxygen radicals. Nitric oxide (NO) can prevent adhesion by decreasing free oxygen radicals due to its superoxide binding properties. Phosphodiesterase inhibitors such as sildenafil citrate increasing NO levels have experimentally shown to inhibit adhesion ${ }^{19}$.

Anti-inflammatory properties of Allium sativum have been previously reported. Allium sativum has been reported to inhibit anti-inflammatory activity by inhibiting activation of nuclear factor kappa-B (NF-kB) caused by oxidative stress ${ }^{20}$. In addition, it is reported in the literature that allium sativum inhibits the production of Thelper 1 cells and inflammatory cytokines, increasing corticosteroid levels, and acts as an anti-inflammatory and immunomodulating agent ${ }^{21}$.

Rassoul et al. ${ }^{22}$, and Kuo et al. ${ }^{23}$ showed that Allium sativum extract showed an anti-inflammatory effect by inhibiting the expression of IL-1 alpha-derived vascular cell adhesion molecule-1 (VCAM-1) and intracellular adhesion molecule-1 (ICAM-1).

In a study by Şahbaz et al. ${ }^{8}$ Allium sativum was shown to be effective in preventing peritoneal adhesion on postoperative day 10 due to its fibrinolytic, antithrombotic, anti-inflammatory and antioxidant effects.

In addition to inflammation and fibrosis, many reactive lymphadenopathies of various sizes were detected in almost all groups. Reactive lymphadenopathy usually occurs due to infections, inflammation or malignancy. Lymph nodes have functions such as microorganism filtration and antibody production. Lymph nodes grow in the presence of microorganisms, malignant cells, or antigenic reactions, which cause the proliferation of lymphocytes or macrophage hyperplasia ${ }^{24}$.

In a study similar to ours, Goret et al. ${ }^{7}$ found in their 2018 study that picnogenol-induced foreign body reaction and lymph node number increased in peritoneal adhesion model. In their study, they reported that the presence of acute inflammation, which later became chronic, played a role in the etiology of lymphoid hyperplasia, which was statistically significant, especially in the group receiving picnogenol.

However, contrary to the results in the literature, we could not detect any positive effects of Allium sativum in addition to daily routine procedures on inflammation, fibrosis or lymphoid hyperplasia. In addition, no benefit of allium sativum was detected in immunohistochemical analysis to detect oxidative stress. 
Although the positive effect of Allium sativum on peritoneal adhesion has been reported in the literature, with the early period findings in our study, it was found that this substance did not provide an additional contribution to the current protocol.

Various studies are needed to test and investigate the effects of this type of traditional medicine used in PA prevention.

\section{- Conclusion}

In this study, no positive results were found that Allium sativum had positive effects on fibrosis, inflammation or reactive lymph node size/number which are among the intraabdominal adhesion parameters; in addition, immunohistochemical analysis to detect oxidative stress at the cellular level did not show a positive decrease in free oxygen radicals.

\section{- References}

1. Menzies $D$, Ellis H. Intestinal obstruction from adhesionshow big is the problem? Ann R Coll Surg Engl. 1990;72(1):603. PMID: 2301905.

2. Coleman MG, McLain AD, Moran BJ. Impact of previous surgery on time taken for incision and division of adhesions during laparotomy. Dis Colon Rectum. 2000;43(9):1297-99. doi: 10.1007/bf02237441.

3. Van Der Krabben AA, Dijkstra FR, Nieuwenhuijzen $M$, Reijnen MM, Schaapveld M, Van Goor H. Morbidity and mortality of inadvertent enterotomy during adhesiotomy. $\mathrm{Br} J$ Surg. 2000;87(4):467-71. doi: 10.1046/j.13652168.2000.01394.x.

4. Kataria H, Singh VP. Liquid paraffin vs hyaluronic acid in preventing intraperitoneal adhesions. Indian J Surg. 2017;79(6):539-43. doi: 10.1007/s12262-016-1522-x.

5. Brochhausen C, Schmitt VH, Planck CN, Rajab TK, Hollemann D, Tapprich C, Krämer B, Wallwiener C, Hierlemann H, Zehbe R, Kirkpatrick JC, Planck, H. Current strategies and future perspectives for intraperitoneal adhesion prevention. J Gastrointest Surg. 2012;16(6):1256-74. doI: 10.1007/s11605-011-1819-9.

6. Sahbaz A, Aynioglu O, Isik H, Gun BD, Cengil O, Erol O, Pycnogenol prevents peritoneal adhesions. Arch Gynecol Obstet. 2015;292(6):1279-84 doi: 10.1007/s00404-0153764-4.

7. Goret CC, Goret NE, Kiraz A, Ozkan OF, Karaayvaz M. The effect of pycnogenol on lymphatic nodes and adhesion during in a peritoneal adhesion model in rats. Acta Cir Bras. 2018;33(2):134-43. doi: 10.1590/s0102865020180020000005.

8. Sahbaz A, Isik H, Aynioglu O, Gungorduk K, Gun BD. Effect of intraabdominal administration of Allium sativum (garlic) oil on postoperative peritoneal adhesion. Eur J Obstet Gynecol Reprod Biol. 2014;177(6):44-7. doi: 10.1016/j. ejogrb.2014.03.018.
9. Zhang YD, YaoW,Wu CX, Chi QM, Zhang JY, Li M. Tropical application of halcinonide cream reduces the severity and incidence of intraperitoneal adhesions in a rat model. Am J Surg2002;184(1):74-7. doi:10.1016/S00029610(02)00876-0.

10. Aldemir $M$, Ozturk $H$, Erten C, Buyukbayram $H$. The preventive effect of Rofecoxib in postoperative intraperitoneal adhesions. Acta Chir Belg. 2004;104(1):97100. doi : 10.1080/00015458.2003.11978403.

11. Hellebrekers BW, Trimbos-Kemper TC, Trimbos JB, Emeis $\mathrm{JJ}$, Kooistra T. Use of fibrinolytic agents in the prevention of postoperative adhesion formation. Fertil Steril. 2000;74(2):203-12. doi: 10.1016/S0015-0282(00)00656-7.

12. Saifzadeh S, Tehrani A, Jalali FSS, Oroujzadeh R. Enhancing effect of aqueous garlic extract on wound healing in the dog: clinical and histopathological studies. J Anim Vet Adv. 2006;5(12):1101-4.

13. Brüggmann $D$, Tchartchian $G$, Wallwiener $M$, Münstedt $K$, Tinneberg HR, Hackethal A. Intra-abdominal adhesions: definition, origin, significance in surgical practice, and treatment options. Dtsch Arztebl Int. 2010;107(44):76975. doi: 10.3238/arztebl.2010.0769.

14. Weibel MA, Mayno G. Peritoneal adhesions and their relationship to abdominal surgery. A postmortem study. Am J Surg. 1973;126(3):345-53. doi: 10.1016/S00029610(73)80123-0.

15. Whang SH, Astudillo JA, Sporn E, Bachman SL, Miedema BW, Davis W, Thaler K. In search of the best peritoneal adhesion model: comparison of different techniques in a rat model. J Surg Res. 2011;167(2):245-50 doi: 10.1016/j. jss.2009.06.020.

16. Alpay Z, Saed GM, Diamond MP. Postoperative adhesions: from formation to prevention. Semin Reprod Med. 2008;26(4):313-21. dol: 10.1055/s-0028-1082389.

17. Fantone JC, Ward PA. Role of oxygen-derived free radicals and metabolites in leukocyte-dependent inflammatory reactions. Am J Pathol. 1982;107(3):395-418. PMID: 6282132.

18. Goret CC, Kiraz A, Goret NE, Kilic SO, Ozkan OF, Karaayvaz $M$. The activity of topical coenzyme Q10 (ubiquinol) in burned rats: Results from an experimental study. East J Med. 2019;24(1):80-5 doi: 10.5505/ejm.2019.66934.

19. Monk BJ, Berman ML, Montz FJ. Adhesions after extensive gynecologic surgery: clinical significance, etiology, and prevention. Am J Obstet Gynecol. 1994;170(5):1396-403. doi: 10.1016/S0002-9378(13)90479-8.

20. Santhosha SG, Prakash Jamuna SN, Prabhavathi. Bioactive components of garlic and their physiological role in health maintenance: a review. Food Biosci. 2013;3(9):59-74 doi: 10.1016/j.fbio.2013.07.001.

21. Hodge G, Hodge S, Han P. Allium sativum (garlic) suppresses leukocyte inflammatory cytokine production in vitro: potential therapeutic use in the treatment of inflammatory bowel disease. Cytometry. 2002;48(4):20915. doi: 10.1002/cyto.10133.

22. Kuo CH, Lee SH, Chen KM, Lii CK, Liu CT. Effect of garlic oil on neutrophil infiltration in the small intestine of endotoxin-injected rats and its association with levels of soluble and cellular adhesion molecules. J Agric Food Chem. 2011;59(14):7717-25. doi:10.1021/jf201185v. 
23. Rassoul F, Salvetter J, Reissig D, Schneider W, Thiery J, Richter V. The influence of garlic (Allium sativum) extract on interleukin 1alpha-induced expression of endothelial intercellular adhesion molecule-1 and vascular cell adhesion molecule-1. Phytomedicine. 2006;13(4):230-5. doi: 10.1016/j.phymed.2005.01.010.

24. Ferrer R. Lymphadenopathy: differential diagnosis and evaluation. Am Fam Physician. 1998;58(6):1313-20. PMID: 9803196.

\section{Correspondence:}

Uğur Topal

Department of General Surgery

Erciyes University Faculty of Medicine

38030 Melilgazi/Kayseri Turkey

Phone: +90 5301139112

sutopal2005@hotmail.com

Received: June 07, 2019

Review: Aug 09, 2019

Accepted: Sept 10, 2019
Conflict of interest: none

Financial source: none

This is an Open Access article distributed under the terms of the Creative Commons Attribution License, which permits unrestricted use, distribution, and reproduction in any medium, provided the original work is properly cited.

${ }^{1}$ Research performed at Health Sciences University Hamidiye Experimental Animal Production and Research Laboratory İstanbul, Turkey. 